\title{
The Impact of Statin Intolerance in Lipid Clinic Patients
}

\author{
Kate Williams ${ }^{1}$, Vinita Mishra ${ }^{2 *}$ \\ ${ }^{1}$ University of Liverpool (Medical School), Liverpool, UK \\ ${ }^{2}$ Clinical Biochemistry and Metabolic Medicine Royal Liverpool and Broadgreen University Hospital NHS Trust, \\ Liverpool, UK \\ Email: "vinmishra@yahoo.co.uk
}

Received 31 March 2015; accepted 17 May 2015; published 20 May 2015

Copyright (C) 2015 by authors and Scientific Research Publishing Inc.

This work is licensed under the Creative Commons Attribution International License (CC BY).

http://creativecommons.org/licenses/by/4.0/

(c) (i) Open Access

\begin{abstract}
Context: Cardiovascular disease is a very common and serious problem in the western world. Statin drug therapy is used in primary, secondary prevention and familial hypercholesterolemia. However, these are frequently associated with adverse effects, causing poor adherence and thus putting patients at risk for future cardiovascular events. Aim: The objective of this study was to review the statin intolerance in lipid patients and to assess the impact of alternative lipid lowering therapy on lipid parameters and cardiovascular outcome in statin intolerant patients. Methodology: 50 patients attending the out-patient lipid clinic of our hospital with statin intolerance were identified. Clinical data on the study patients were gathered retrospectively relating to statin intolerance and the clinical effectiveness of alternative lipid lowering therapy on lipid parameters and cardiovascular outcome. Results: Rosuvastatin was the most intolerable whereas pravastatin or fluvastatin was the most tolerable statin in our study patients. Myalgia was the commonly reported adverse effect of statin. The low dose statin monotherapy or combination of low dose statin and ezetemibe was the most tolerable alternative lipid lowering therapy in statin intolerant patients. After an average period of 10 months of initiation of alternative lipid lowering therapy; combination of low dose statin plus ezetimibe showed the largest reduction in serum total cholesterol and low-density lipoprotein (LDL) cholesterol levels. Conclusions: Pravastatin should be preferred in statin intolerant patients. A combination of low dose statin plus ezetimibe appeared to be the most tolerable and clinically effective therapy in statin intolerant patients.
\end{abstract}

\section{Keywords}

Statin Intolerance, Alternative Lipid Lowering Therapy, Low-Density Lipoprotein (LDL), Total Cholesterol

${ }^{*}$ Corresponding author. 


\section{Introduction}

Cardiovascular disease is associated with significant mortality and morbidity worldwide; it is the most prevalent medical condition in Western societies [1]. Each year over 4.3 million people die due to cardiovascular diseases in Europe, causing nearly half of all deaths (48\%) [1]. Multiple risk factors, including hypertension, diabetes mellitus, dyslipidaemia, sedentary lifestyle, smoking and stress potentiate cardiovascular morbidity [2]. Cardiovascular risk is directly related to serum total cholesterol levels [3], with the World Health Report 2002 estimating $>60 \%$ of coronary heart disease and $40 \%$ of ischaemic stroke in developed countries is due to raised serum total cholesterol levels [1].

Management of hypercholesterolemia includes lifestyle changes such as smoking cessation, healthy diet, weight loss and regular exercise [3]; alongside which pharmacological intervention may be required. Statins are the most commonly and successfully used drugs for lowering serum total cholesterol [4], and have been shown to be effective in primary prevention (no previous cardiovascular events), secondary prevention (previous cardiovascular event) and familial hypercholesterolemia [4] [5]. Statins mainly work through lowering low-density lipoprotein (LDL)-cholesterol, however, they are also thought to reduce vascular inflammation and thrombus formation, as well as improving endothelial function [6]. Hence use of statins produces significant reduction in cardiovascular morbidity and overall mortality in primary and secondary prevention populations [6].

Nevertheless, statin use is associated with adverse effects including raised liver enzymes, muscle problems (myalgia, myositis, rhabdomyolysis), transient proteinuria, sleep problems, sexual dysfunction, renal failure, glucose elevations, neurological problems (haemorrhagic stroke, cognitive decline, peripheral neuropathy), depression and alopecia [7]. Studies have reported 18.2\% side effects with statins; leading to reduced patient adherence and loss of risk reducing effects [7]. Recently studies have suggested a link between statin uses and developing diabetes mellitus; they however concluded that risk is low in both absolute terms when compared with reduction in coronary events [8]. Currently the reason for this increased risk of developing diabetes is poorly understood [9].

When patients with primary or secondary prevention and familial hypercholesterolemia develop statin intolerance continuation of their lipid-lowering therapy, it is essential to reduce their cardiovascular risk. According to American College of Cardiology and American Heart Association (2013) recommendation [10], clinicians should consider "moderated” therapy with combination of low intensity statin and another lipid modifying medications among high-risk patients who are intolerant or unresponsive to high intensity statin. There are several therapeutic options that may be considered, including the use of alternative or low dose statin, either alone or in addition to non-statin lipid lowering therapies [11]. Ezetimibe, niacin, fibrates, bile acid sequestrants, omega-3 fatty acid and LDL apheresis are all examples of non-statin lipid lowering therapies. They all work in different ways to statins and each other, and thus have different effectiveness. Ezetimbe acts by directly inhibiting the cholesterol transporter NPC1L1. Niacin decreases the hepatic secretion of VLDL (very low density lipoprotein) and lowers free fatty-acid mobilization from the periphery; it has been shown to lower both LDL cholesterol, triglycerides and increase HDL (high density lipoprotein) cholesterol levels. Fibrates lower triglyceride and raise HDL cholesterol. Bile acid sequestrants bind bile acids in the intestine and prevent their enterohepatic recirculation, indirectly lowering LDL cholesterol. Omega-3 fatty acid is useful in patients with high triglycerides. LDL apheresis is a method used for very high-risk patients who have not responded to other treatments. It selectively removes LDL from either plasma or whole blood, lowering LDL cholesterol levels by $50 \%$ - $75 \%$ [11].

The aim of this study was to review the statin intolerance in our lipid clinic patients, assessing the tolerability and clinical effectiveness of alternative lipid lowering therapy in statin intolerant patients on lipid parameters (total cholesterol and LDL cholesterol) and cardiovascular outcome.

\section{Methodology}

\subsection{Study Design and Setting}

50 patients attending the lipid outpatient clinic with statin intolerance were identified from clinic letters. The data on statin intolerance was collected including statin dose, length of time (before intolerance presented) and side effects experienced. 


\subsection{Study Population}

The statin intolerant patients were categorised into primary prevention, secondary prevention and familial hypercholesterolemia. The alternative lipid lowering therapy, which was either alternative low dose statin only, combination of statin with non-statin lipid modifying medication or only non-statin therapy was initiated in patients after they presented with statin intolerance.

\subsection{Data Analysis}

Impact of alternative lipid lowering therapy in statin intolerant patients (study cohort) was analysed by calculating the absolute reduction (\%) in serum total cholesterol, LDL-cholesterol after the introduction of this change in lipid lowering therapy. Besides this data on occurrence of cardiovascular events during the therapy was also collected to assess the clinical effectiveness of alternative lipid lowering therapy in statin intolerant patients.

\section{Results}

\subsection{Basic Data}

Statin intolerant patients $(n=50)$ were being treated for primary prevention $(n=29)$, secondary prevention $(n=$ 15) and familial hypercholesterolemia $(n=6)$, with an average age of 59.2 years (range 31 - 90); consisting of 30 women (average age 64.1 years, range 49 - 90) and 20 men (average age 54.4 years, range 31 - 78).

\subsection{Data of Statin Intolerance (Table 1)}

In this cohort of 50 patients; intolerance to rosuvastatin $(n=19)$, simvastatin $(n=17)$, atorvastatin $(n=16)$, pravastatin $(n=9)$, fluvastatin $(n=1)$ and intolerance to more than one statin $(n=11)$.

\subsubsection{Intolerance to Rosuvastatin}

13 female, 6 male, average age 58.5 years. 1 patient diagnosed with familial hypercholesterolemia, 6 patients were with secondary prevention and 12 patients were on rosuvastatin for primary prevention. Intolerance was caused by doses ranging from $5 \mathrm{mg}$ to $40 \mathrm{mg}$ (patients; $\mathrm{n}=9$ on $5 \mathrm{mg} ; \mathrm{n}=8$ on $10 \mathrm{mg} ; \mathrm{n}=1$ on $20 \mathrm{mg} ; \mathrm{n}=1$ on $40 \mathrm{mg}$ ). The length of time before discontinuing due to intolerance varied from 3 months to 3 years with no apparent relationship between this and dose. Muscle aches were the most common side effect experienced. None of the patients developed diabetes mellitus.

Table 1. Overview of statin intolerance in statin intolerant patients.

\begin{tabular}{|c|c|c|c|c|c|}
\hline Statin & $\begin{array}{l}\text { Number of } \\
\text { intolerant } \\
\text { Patients }\end{array}$ & $\begin{array}{c}\text { Most common } \\
\text { dose }\end{array}$ & $\begin{array}{l}\text { Average length } \\
\text { of time before } \\
\text { intolerance }\end{array}$ & Side effects experienced & $\begin{array}{l}\text { Most common side } \\
\text { effect (number) }\end{array}$ \\
\hline Simvastatin & 17 & $40 \mathrm{mg}$ & 7.6 months & $\begin{array}{l}\text { Muscle problems, raisedcreatine } \\
\text { kinase, headache, fatigue, joint pain, } \\
\text { Itching, eczema, neuropathy, sleep } \\
\text { disturbance, Depression }\end{array}$ & Muscle aches (6) \\
\hline Atorvastatin & 16 & $40 \mathrm{mg}$ & 8.25 months & $\begin{array}{l}\text { Muscle problems, raisedcreatine } \\
\text { kinase, joint pain, Itching, neuropathy, } \\
\text { decreased appetite, abdominal pains, } \\
\text { raised liver function tests }\end{array}$ & Muscle aches (8) \\
\hline Pravastatin & 9 & $10 \mathrm{mg}$ & 14 months & $\begin{array}{l}\text { Muscle problems, itching, } \\
\text { nightmares, urinary problems }\end{array}$ & Muscle aches (5) \\
\hline Rosuvastatin & 19 & $5 \mathrm{mg}$ & 5.8 months & $\begin{array}{l}\text { Muscle problems, fatigue, } \\
\text { joint pain, memory loss, } \\
\text { gastrointestinal bleed, diarrhoea }\end{array}$ & Muscle aches (13) \\
\hline Fluvastatin & 1 & $10 \mathrm{mg}$ & 6 months & Gastrointestinal problem & Gastrointestinal problem \\
\hline
\end{tabular}




\subsubsection{Intolerance to Simvastatin}

12 females, 5 males, average age 60.5 years. 2 patients diagnosed with familial hypercholesterolemia, 2 patients were with secondary prevention and 13 patients with primary prevention. Intolerance was caused by doses ranging from $10 \mathrm{mg}$ to $80 \mathrm{mg}$ (patients; $\mathrm{n}=4$ on $10 \mathrm{mg} ; \mathrm{n}=5$ on $20 \mathrm{mg} ; \mathrm{n}=7$ on $40 \mathrm{mg} ; \mathrm{n}=1 \mathrm{on} 80 \mathrm{mg}$ ). The length of time before discontinuing due to intolerance varied from 2 weeks to 3 years, with no apparent relationship between this and dose. A wide variety of side effects were experienced, with the most common being muscle aches. None of the patient developed diabetes mellitus whilst on simvastatin.

\subsubsection{Intolerance to Atorvastatin}

7 female, 9 male, average age 62.6 years. 4 patients diagnosed with familial hypercholesterolemia, 5 patients were with secondary prevention and 7 patients were on atorvastatin for primary prevention. Intolerance was caused by doses ranging from $10 \mathrm{mg}$ to $80 \mathrm{mg}$ (patients; $\mathrm{n}=3$ on $10 \mathrm{mg} ; \mathrm{n}=2$ on $20 \mathrm{mg} ; \mathrm{n}=7$ on $40 \mathrm{mg} ; \mathrm{n}=1$ on $60 \mathrm{mg}$ and $\mathrm{n}=3 \mathrm{on} 80 \mathrm{mg}$ ). The length of time before discontinuing due to intolerance varied from 2 months to 2.5 years with no apparent relationship between this and dose. Muscle aches again being the most common side effects experienced. One patient developed impaired glucose tolerance whilst on $40 \mathrm{mg}$ atorvastatin after 12 months.

\subsubsection{Intolerance to Pravastatin}

5 female, 4 male, average age 59.6 years. 1 patient diagnosed with familial hypercholesterolemia, 4 patients with secondary prevention and 4 patients on pravastatin for primary prevention. Intolerance was caused by doses ranging from $10 \mathrm{mg}$ to $40 \mathrm{mg}$ (patients $\mathrm{n}=7 \mathrm{on} 10 \mathrm{mg}$; $=1$ on $20 \mathrm{mg} ; \mathrm{n}=1 \mathrm{on} 40 \mathrm{mg}$ ). The length of time before discontinuing due to intolerance varied from 3 months to 3 years with no apparent relationship between this and dose. The most common side effect experienced was muscle aches. One patient developed diabetes mellitus whilst on $10 \mathrm{mg}$ pravastatin for 18 months.

\subsubsection{Intolerance to Fluvastatin}

One 52-year-old male developed intolerance to fluvastatin $10 \mathrm{mg}$ whilst being treated for secondary prevention; stopping due to gastrointestinal problems after 6 months.

\subsubsection{Intolerant to More Than One Statin}

11 patients were intolerant to multiple statins, with one patient intolerant to 3 statins (atorvastatin, rosuvastatin and pravastatin); the rest being intolerant to 2 statins. Majority patients $(n=8)$ were intolerant to rosuvastatin.

\subsection{Impact of Alternative Lipid Lowering Therapy in Statin Intolerant Patients}

The patients intolerant to statins were commenced on alternative lipid lowering therapy, which was either low dose alternative statin only, combination of low dose alternative statin with non-statin lipid modifying therapy or only non-statin lipid modifying therapy. The data was collected with respect to total cholesterol, LDL cholesterol, and new cardiovascular eventafter an average period of ten months of starting the alternative lipid lowering therapy.

\subsubsection{Impact on Serum Total Cholesterol and LDL-Cholesterol (Table 2)}

\section{Alternative statin only therapy $(n=15)$}

Overall reduction in both total cholesterol and LDL cholesterol was seen on statin only therapy $(13 \%, 17 \%$ respectively). 1 patient was on atorvastatin (10 mg for 4 months), 4 patients on pravastatin (10 - $40 \mathrm{mg}$ for average 9.5 months), 10 patients were on rosuvastatin (patients $n=9$ on $5 \mathrm{mg}, \mathrm{n}=1$ on $20 \mathrm{mg}$ for an average of 11 months). Pravastatin had a reducing effect on all 4 patients' lipids, with an average reduction of $23 \%$ in total cholesterol and 33\% reduction in LDL-cholesterol. Those on rosuvastatin had an average reduction in both total cholesterol (10\%) and LDL-cholesterol (14\%).

\section{Alternative statin plus non-statin lipid lowering therapy $(n=25)$}

Majority of the patients $(\mathrm{n}=19)$, were on a low dose statin (rosuvastatin 5 - $10 \mathrm{mg}$ daily was the most commonly used statin) plus non-statin lipid lowering drug (ezetimibe, fibrate, bile acid sequestrant). Patients on statin plus ezetemibe $(n=15)$ showed a reduction in both their total cholesterol and LDL-cholesterol with average 
Table 2. Impact of alternative lipid lowering therapy on lipid parameters and cardiovascular outcome.

\begin{tabular}{|c|c|c|c|c|c|c|}
\hline \multirow{2}{*}{\multicolumn{2}{|c|}{ Alternative lipid lowering therapy }} & \multirow{2}{*}{$\begin{array}{l}\text { Number } \\
\text { of Patients }\end{array}$} & \multicolumn{2}{|c|}{ Absolute change in lipid parameters } & \multirow{2}{*}{$\begin{array}{l}\text { Average duration } \\
\text { of therapy } \\
\text { (Months) }\end{array}$} & \multirow{2}{*}{$\begin{array}{l}\text { Cardiovascular } \\
\text { event during } \\
\text { therapy }\end{array}$} \\
\hline & & & $\begin{array}{c}\text { Total Cholesterol } \\
\text { (\%) }\end{array}$ & $\begin{array}{l}\text { LDL-cholesterol } \\
\text { (\%) }\end{array}$ & & \\
\hline Alternative statin only & Low dose statin & 15 & -10.3 & -14 & 10.2 & 1 \\
\hline \multirow{4}{*}{$\begin{array}{l}\text { Alternative statin } \\
\text { plus non-statin lipid } \\
\text { lowering therapy }\end{array}$} & Statin plus ezetemibe & 15 & -28.5 & -37.3 & 10.9 & 1 \\
\hline & Statin plus fibrate & 3 & -11.2 & -16.2 & 6 & 0 \\
\hline & $\begin{array}{l}\text { Statin plus bile acid } \\
\text { sequestrant }\end{array}$ & 1 & 0 & +2.2 & 10.0 & 0 \\
\hline & $\begin{array}{l}\text { Statin plus more than } \\
\text { one non-statin therapy }\end{array}$ & 6 & -16 & -4 & 9.8 & 0 \\
\hline \multirow{4}{*}{$\begin{array}{l}\text { Non-statin lipid } \\
\text { lowering therapy only }\end{array}$} & Ezetemibe & 3 & +17.7 & +21.7 & 9.6 & 0 \\
\hline & Fibrate & 2 & -29 & -32.1 & 10.5 & 0 \\
\hline & Bile acid sequestrant & 2 & +5.5 & 0 & 11 & 0 \\
\hline & Omega 3 fatty acid & 3 & +5.1 & +25 & 15.5 & 0 \\
\hline
\end{tabular}

reductions of $28.5 \%$ and $37.3 \%$ respectively after an average of 10.9 months. Three patients were on statin plus fibrate combination and showed an average reduction in total cholesterol (11.2\%) and LDL-cholesterol (16.2\%) after an average 6 months on this combination. Only one patient was on statin plus bile acid sequestrant and showed no change in their total cholesterol but an increase in LDL-cholesterol (2.2\%). Six patients were taking a statin plus multiple non-statin lipid lowering therapies and showed a reduction in both total and LDL-cholesterol (16\% and $4 \%$ respectively) after 10 months on average.

\section{Non-statin lipid lowering therapy only $(n=10)$}

A non-statin lipid lowering therapy included ezetimibe, fibrates, bile acid sequestrants, and omega 3 fatty acid. Only 2 of these patients on fenofibrate showed any reduction in their total cholesterol level, the rest having either no change at all or an increase in total cholesterol.

\subsubsection{Impact on Cardiovascular Outcome}

One patient experienced a myocardial infarction and underwent a coronary artery bypass graft whilst on a combination of pravastatin $40 \mathrm{mg}$ and ezetimibe $10 \mathrm{mg}$. Another patient who was only on pravastatin $40 \mathrm{mg}$ also had myocardial infarction whilst on this medication. Both these patients were on lipid lowering therapy for primary prevention.

\section{Discussion}

Recent years have seen tremendous increase in statin prescription [12]. Further rise is expected with introduction of new guidance JBS 3 (Joint British Societies' consensus recommendations for the prevention of cardiovascular disease) [13] and NICE 2014 (National institute for health and care excellence) [14], availability of generic statins and increasing use in younger population. Nonetheless, increasing use of statins may result in poor compliance due to its adverse effects particularly on muscles. Statins are associated wide clinical spectrum of myopathy encompassing myalgia, myositis, rahbdomyolysis and an asymptomatic increase in creatine kinase. Myalgia was the most common cause of statin intolerance in our study patients as they presented with muscle aches and normal creatine kinase. This is consistent with results of randomised controlled trials and clinical trials where at least one fourth of participants presented with myalgia [15]. In clinical practice muscle related adverse effect with statin use in patients has been reported to be as high as 1.2 per 10,000 person years [16].

Several risk factors have been proposed to potentiate the statin related adverse effect on muscles. This includes, dose and intensity of statin, advanced age, gender particularly female sex, low body mass index and 
concomitant medications. There were more women $(n=30)$ than men $(n=20)$ in our study group; with the female group having an average age nearly 10 years older than that of the male group. The statin intolerance was seen with all doses and intensity of statins in our study patients. It has been suggested that statin dose may be responsible for muscle effect by increasing the serum concentration of statin, nevertheless no link has been seen between intramuscular statin concentration and myopathy [17]. Studies have shown that lipophilic statins (atorvastatin, simvastatin) are more likely to result into muscle problems than hydrophilic statins such as pravastatin, fluvastatin and rosuvastatin [18]. This is because lipophilic statins infiltrate muscles and can have myotoxic effects. In our study patients, rosuvastatin was the most intolerant statin, generally resulting in muscle problems seen even at low dose of $5 \mathrm{mg}$ daily. Simvastatin and atorvastatin both at $40 \mathrm{mg}$ daily dose were also reasonably common causes of intolerance; with muscle aches being the most prevalent side effect. Pravastatin and fluvastatin were less common causes of statin intolerance with respect to myalgia in our study cohort. Observational studies have shown that pravastatin is least known to cause myalgia amongst all statins [19]. The length of time before statin intolerance occurred varied greatly within the cohort; however for all statins involved, the average time until intolerance to statin was nearly 6 months; with pravastatin having the longest time until intolerance at an average of 14 months. This is consistent with recent epidemiological study performed in England and Wales where statin related adverse effects appeared within a year of statin use [20]. Only 2 patients in this cohort developed changes in their glucose control; one developing impaired glucose tolerance on atorvastatin $40 \mathrm{mg}$ daily for 12 months, the other developing diabetes mellitus on pravastatin $10 \mathrm{mg}$ daily for 18 months. Food and Drug Administration recently added information to statin label that its use increases the serum glucose and therefore increases the risk of diabetes mellitus in patients on statin [21]. Nevertheless the cardiovascular benefits of statins outweigh its glycaemic effect and thus statin use is prudent for public health.

Statin intolerance can be managed by changing either the dose or type of statin or through using other nonstatin lipid lowering therapy such as ezetimibe, fibrates, omega-3 fatty acid and bile acid sequestrants. Our study patients who were intolerant to statins were able to tolerate either alternative low dose statin only or combination of alternative low dose statin plus ezetimibe. The most tolerable alternative lipid lowering therapy in our cohort of patients waseither low dose rosuvastatin (5 - $10 \mathrm{mg}$ daily) or pravastatin monotherapy or in combination with ezetemibe.

We assessed the impact of this lipid lowering therapy by calculating the change in total cholesterol and LDL cholesterol after starting the alternative lipid lowering therapy in statin intolerant patients. Recent meta-analysis has shown that reduction in LDL cholesterol by 2 - $3 \mathrm{mmol} / \mathrm{L}$ reduces the cardiovascular risk by $40 \%$ - 50\% [22]. The maximum reduction was observed with combination of alternative low dose statin and ezetemibe over an average duration of 10.9 months (Table 2). There were only four patients who were on combination of alternative statin and fibrate or fibrate only and all these patients showed reduction in total cholesterol and LDL cholesterol. However, statin in combination with bile acid sequestrants, ezetimibe only, bile acid sequestrants only and omega-3 fatty acid showed least reduction or an increase in total cholesterol and LDL cholesterol levels. Systematic review of 36 clinical trials have shown similar results where low intensity statin in combination with non-statin therapy results in reduction in LDL cholesterol [23] but has insufficient evidence for non-statin monotherapy.

The lipid lowering therapy in statin intolerant patients resulted in reduction in total cholesterol and LDL cholesterol after an average period of ten months (Table 3) in all three categories(primary or secondary prevention and familial hypercholesterolemia). This was reflected in clinical outcome as only two patients presented with myocardial infarction whilst on lipid lowering therapy; both undergoing treatment for primary prevention; one of which was on pravastatin only and the other pravastatin plus ezetimibe.

Table 3. Average reduction (\%) in serum total cholesterol and LDL cholesterol levels after starting alternative lipid lowering therapy in statin intolerant patients.

\begin{tabular}{cccc}
\hline & Number of patients & $\begin{array}{c}\text { Average reduction in total } \\
\text { cholesterol (\%) }\end{array}$ & $\begin{array}{c}\text { Average reduction in } \\
\text { LDL-Cholesterol (\%) }\end{array}$ \\
\hline Primary Prevention & 29 & 16 & 19 \\
Secondary Prevention & 15 & 13.2 & 22.2 \\
Familial Hypercholesterolemia & 6 & 13.2 & 13 \\
\hline
\end{tabular}




\section{Study Limitations}

There are limitations to our study as it is a small observational study of shorter duration. Further pragmatic long term clinical trials are required to determine the most clinically effective alternative lipid lowering therapy in patients intolerant to statins.

\section{Conclusion}

Our study suggests that alternative lipid lowering therapy in statin intolerant patients is able to reduce total cholesterol and LDL cholesterol. Pravastatin is the most tolerant statin whereas rosuvastatin is the most intolerant statin. Combination of low dose statin (pravastatin or rosuvastatin) and ezetemibe is clinically effective and tolerant alternative lipid lowering therapy in statin intolerant patients. Non-statin lipid lowering monotherapy is clinically ineffective in statin intolerant patients

\section{References}

[1] British Heart Foundation (2008) European Cardiovascular Disease Statistics. London.

[2] Ferdinand, K.C. (2011) Are Cardiovascular Benefits in Statin Lipid Affects Dependent on Baseline Lipid Levels? Current Atherosclerosis Reports, 13, 64-72. http://dx.doi.org/10.1007/s11883-010-0149-9

[3] Steingo, L. (1993) Epidemiology of Hyperlipidemia and the Efficacy of Pravastatin Therapy. Current Therapeutic Research-Clinical and Experimental, 54, 290-299. http://dx.doi.org/10.1016/S0011-393X(05)80629-0

[4] Yusuf, P.S., Hawken, S., Ôunpuu, S., Dans, T., Avezum, A. and Lanas, F. (2004) Effect of Potentially Modifiable Risk Factors Associated with Myocardial Infarction in 52 Countries (the INTERHEART Study): Case-Control Study. The Lancet, 364, 937-952. http://dx.doi.org/10.1016/S0140-6736(04)17018-9

[5] Gotto Jr., A.M. and Moon, J.E. (2012) Management of Cardiovascular Risk: The Importance of Meeting Lipid Targets. American Journal of Cardiology, 110, 3A-14A. http://dx.doi.org/10.1016/j.amjcard.2012.04.002

[6] Minder, C.M., Blaha, M.J., Horne, A., Michos, E.D., Kaul, S. and Blumenthal, R.S. (2012) Evidence-Based Use of Statins for Primary Prevention of Cardiovascular Disease. American Journal of Medicine, 125, 440-446. http://dx.doi.org/10.1016/j.amjmed.2011.11.013

[7] NICE (2010) Lipid Modifications: Cardiovascular Risk Assessment and the Modification of Blood Lipids for the Primary and Secondary Prevention of Cardiovascular Disease. UK

[8] Alberton, M., Wu, P., Druyts, E., Briel, M. and Mills, E.J. (2012) Adverse Events Associated with Individual Statin Treatments for Cardiovascular Disease: An Indirect Comparison Meta-Analysis. QJM, 105, 145-157. http://dx.doi.org/10.1093/qjmed/hcr158

[9] Mills, E.J., Wu, P., Chong, G., Ghement, I., Singh, S., Akl, E.A., Eyawo, O., Guyatt, G., Berwanger, O. and Briel, M.(2011) Efficacy and Safety of Statin Treatment for Cardiovascular Disease: A Network Meta-Analysis of 170,255 Patients from 76 Randomized Trials. QJM, 104, 109-124. http://dx.doi.org/10.1093/qjmed/hcq165

[10] Stone, N.J., Robinson, J., Lichtenstein, A.H., Merz, C.N., Blum, C.B., Eckel, R.H., et al. (2013) ACC/AHA Guideline on the Treatment of Blood Cholesterol to Reduce Atherosclerotic Cardiovascular Risk in Adults: A Report of the American College of Cardiology/American Heart Association Task Force on Practice Guidelines. Circulation, 129, S1S45.

[11] Mancini, G.B.J., Baker, S., Bergeron, J., Fitchett, D., Frohlich, J., Genest, J., Gupta, M., Hegele, R.A., Ng, D. and Pope, J. (2011) Diagnosis, Prevention, and Management of Statin Adverse Effects and Intolerance: Proceedings of a Canadian Working Group Consensus Conference. Canadian Journal of Cardiology, 27, 635-662. http://dx.doi.org/10.1016/j.cjca.2011.05.007

[12] Law, M. and Rudnicka, A.R. (2006) Statin Safety: A Systematic Review. American Journal of Cardiology, 97, S52S60. http://dx.doi.org/10.1016/j.amjcard.2005.12.010

[13] JBS3 Board (2014) Joint British Societies' Consensus Recommendations for the Prevention of Cardiovascular Disease (JBS3). Heart, 100, ii1-ii67.

[14] NICE (2014) Lipid Modification: Cardiovascular Risk Assessment and the Modification of Blood Lipids for the Primary and Secondary Prevention of Cardiovascular Disease. NICE, UK.

[15] Bays, H. (2005) Statin Safety: An Overview and Assessment of the Data. American Journal of Cardiology, 97, S6-S26. http://dx.doi.org/10.1016/j.amjcard.2005.12.006

[16] Gaist, D., Rodríguez, L.A., Huerta, C., Hallas, J. and Sindrup, S.H. (2001) Lipid-Lowering Drugs and the Risk of Myopathy: A Population-Based Follow-Up Study. Epidemiology, 12, 565-569.

http://dx.doi.org/10.1097/00001648-200109000-00017 
[17] Thompson, P.D., Clarkson, P.M. and Rosenson, R.S. (2006) An Assessment of Statin Safety by Muscle Experts. American Journal of Cardiology, 97, S69-S76. http://dx.doi.org/10.1016/j.amjcard.2005.12.013

[18] Rosenson, R.S. (2004) Current Overview of Statin-Induced Myopathy. American Journal of Medicine, 116, $408-416$. http://dx.doi.org/10.1016/j.amjmed.2003.10.033

[19] Keogh, A., Macdonald, P., Kaan, A., Aboyoun, C., Spratt, P. and Mundy, J. (2000) Efficacy and Safety of Pravastatin versus Simvastatin after Cardiac Transplantation. Journal of Heart and Lung Transplantation, 19, 529-537. http://dx.doi.org/10.1016/S1053-2498(00)00077-2

[20] Hippisley-Cox, J. (2010) Unintended Effects of Statins in Men and Women in England and Wales: Population Based Cohort Study Using the QResearch Database. BMJ, 340, c2197. http://dx.doi.org/10.1136/bmj.c2197

[21] Carter, A.A., Gomes, T., Camacho, X., Juurlink, D.N., Shah, B.R. and Mamdani, M.M. (2013) Risk of Incident Diabetes among Patients Treated with Statins: Population Based Study. BMJ, 346, f2610. http://dx.doi.org/10.1136/bmj.f2610

[22] Cholesterol Treatment Trialists' (CTT) Collaboration (2010) Efficacy and Safety of More Intensive Lowering of LDL Cholesterol: A Meta-Analysis of Data from 170000 Participants in 26 Randomised Trials. Lancet, 376, 1670-1681. http://dx.doi.org/10.1016/S0140-6736(10)61350-5

[23] Kimberly, A.G., Monroe, A.K., Sharma, R., Ranasinghe, P.D., Chelladurai, Y. and Robinson, K.A. (2014) Effectiveness of Combination Therapy with Statin and Another Lipid-Modifying Agent Compared with Intensified Statin Monotherapy. Annals of Internal Medicine, 160, 468-476. http://dx.doi.org/10.7326/M13-2526 